\title{
Acute Pancreatitis as a Possible Unusual Manifestation of COVID-19 in Children
}

\author{
Niloufar Bineshfar (D), ${ }^{1}$ Alireza Mirahmadi $\left(\mathbb{D},{ }^{1}\right.$ Fereshteh Karbasian (D), \\ Elham Pourbakhtyaran $\left(\mathbb{D},{ }^{3}\right.$ Abdollah Karimi $\mathbb{D}^{4},{ }^{4}$ and Mehdi Sarafi $\mathbb{D}^{5}$ \\ ${ }^{1}$ Student Research Committee, School of Medicine, Shahid Beheshti University of Medical Sciences, Tehran, Iran \\ ${ }^{2}$ Department of Pediatrics Emergency, Mofid Children's Hospital, Shahid Beheshti University of Medical Sciences, Tehran, Iran \\ ${ }^{3}$ Pediatric Specialist, Emergency Department, Shahid Beheshti University of Medical Sciences, Tehran, Iran \\ ${ }^{4}$ Pediatric Infections Research Center, Mofid Children's Hospital, Shahid Beheshti University of Medical Sciences, Tehran, Iran \\ ${ }^{5}$ Pediatric Surgery Research Center, Research Institute for Children's Health, Shahid Beheshti University of Medical Sciences, \\ Tehran, Iran
}

Correspondence should be addressed to Fereshteh Karbasian; karbasian.md@sbmu.ac.ir

Received 30 November 2020; Revised 30 December 2020; Accepted 24 January 2021; Published 3 February 2021

Academic Editor: Maria Moschovi

Copyright (c) 2021 Niloufar Bineshfar et al. This is an open access article distributed under the Creative Commons Attribution License, which permits unrestricted use, distribution, and reproduction in any medium, provided the original work is properly cited.

Coronavirus disease-2019 (COVID-19) which is caused by severe acute respiratory syndrome coronavirus-2 (SARS-CoV-2) has spread throughout the world causing problems for millions of people. Symptoms of COVID-19 in pediatric patients include both respiratory and gastrointestinal symptoms. The most common symptoms are fever, cough, and fatigue. In this report, we describe a case of a previously well 14-year-old boy, who presented to our emergency department with a complaint of abdominal pain, nausea, and vomiting without fever or respiratory symptoms. He was diagnosed with acute pancreatitis based on an abnormal amylase level and abdomen computed tomography (CT) and later found to be infected by SARS-CoV-2, by a positive reverse transcriptase-polymerase chain reaction (RT-PCR) test.

\section{Introduction}

In December 2019, severe acute respiratory syndrome coronavirus-2 (SARS-CoV-2), which causes coronavirus disease-2019 (COVID-19), was discovered in Wuhan, China [1].

COVID-19 in children presented mostly with mild upper respiratory symptoms. The most common symptoms are fever, cough, and fatigue; however, gastrointestinal symptoms such as diarrhea, vomiting, and abdominal pain were also reported $[2,3]$.

Acute pancreatitis (AP) which is caused by medications, trauma, gallstones, and less prevalently viral infections in pediatric patients is the sudden inflammation of the pancreas $[4,5]$. We report a case of a COVID-19associated pancreatitis presenting in a 14 -year-old boy.

\section{Case Report}

A 14-year-old boy presented to our emergency department with abdominal pain in the epigastric region associated with anorexia, nausea, and vomiting for 24 hours. The patient had no fever or respiratory symptoms. There was no underlying disease. He mentioned a history of contact with his aunt who was suspected of COVID-19. On physical examination, the patient was not ill or toxic and vital signs were normal: RR: 20, PR: 98, BP: $100 / 65$, and $\mathrm{T}: 36.9^{\circ} \mathrm{C}$. The abdomen was soft, nondistended with right lower quadrant and epigastric tenderness.

Laboratory tests revealed elevated amylase (1914, normal $<100 \mu / \mathrm{l})$ and lymphopenia, and other blood test results were within normal limits (Table 1). Later, a nasopharyngeal swab specimen was collected which tested positive for SARS-CoV-2 on reverse transcriptase-polymerase chain reaction (RT-PCR). 
TABLE 1: Laboratory results.

\begin{tabular}{|c|c|c|c|}
\hline Laboratory results & Day 1 & Day 2 & Day 3 \\
\hline White cell count & 23.9 & 9.3 & 10.3 \\
\hline \multicolumn{4}{|l|}{ Differential count } \\
\hline Neutrophils & 22466 & 7877 & 8034 \\
\hline Lymphocytes & 956 & 912 & 1545 \\
\hline Hemoglobin & 13.9 & 9.6 & 11.7 \\
\hline Hematocrit & 41.4 & 30.5 & 36.4 \\
\hline Platelet count & 369 & 184 & 217 \\
\hline ALT & 38 & 20 & \\
\hline AST & 33 & 15 & \\
\hline Alkaline phosphatase & 328 & 232 & \\
\hline Amylase & 1914 & 200 & \\
\hline Blood sugar & 120 & & 94 \\
\hline Blood urea nitrogen & 14.3 & & 5.6 \\
\hline Creatinine & 0.8 & & 0.56 \\
\hline C-reactive protein & 4 & 4 & 11 \\
\hline $\mathrm{Na}$ & 140 & & 138 \\
\hline K & 4.5 & & 4.5 \\
\hline Erythrocyte sedimentation rate & 18 & 26 & 42 \\
\hline
\end{tabular}

Due to the positive SARS-CoV-2 RT-PCR, a chest X-ray (Figure 1) and computed tomography (CT) (Figure 2) were obtained. Abdomen CT was suggestive of pancreatitis (Figure 3).

The patient was treated with bowel rest, intravenous crystalloid fluid resuscitation, and ondansetron, pantoprazole, and empiric antibiotics including ceftriaxone and metronidazole. The symptoms were resolved gradually within 3 days, and the patient was discharged with decreasing amylase level.

\section{Discussion}

AP in pediatric patients is most commonly caused by drugs, trauma, and gallstones $[4,5]$. Less common causes of AP have been reported as infections. The relation between AP and some viruses such as CMV, HIV, HSV, EBV, VZV, mumps virus, coxsackievirus, and some others has been proven [4-7]. Based on the Revised Atlanta Classification System for AP Diagnosis, at least two of the three following criteria should be found: (1) abdominal pain (defined as acute onset, persistent, severe epigastric pain often radiating to the patients' back), (2) increased serum lipase or amylase levels to greater than 3 times the upper limit of normal value, and (3) characteristic findings of AP on contrast-enhanced CT [8]. AP was diagnosed in our patient based on the Atlanta criteria. In the search for etiology, none of the common causes of AP was found and the only significant finding was a positive SARS-CoV-2 PCR test. Recent studies have shown gastrointestinal symptoms are fairly common in COVID-19 but the relation between COVID-19 and AP has not been described [9-11]. However, a case series of 52 COVID-19 patients reported pancreas injury in 9 patients $(17 \%$ of patients) [12]. The clear pathogenesis of AP in COVID-19 patients is unknown, but the mechanism of AP following viral infections is different and depends on the type of the virus involved [5]. Currently, there is some evidence showing SARS$\mathrm{CoV}-2$ enters cells in the lungs and gastrointestinal system by binding to the angiotensin-converting enzyme 2 (ACE2) (and

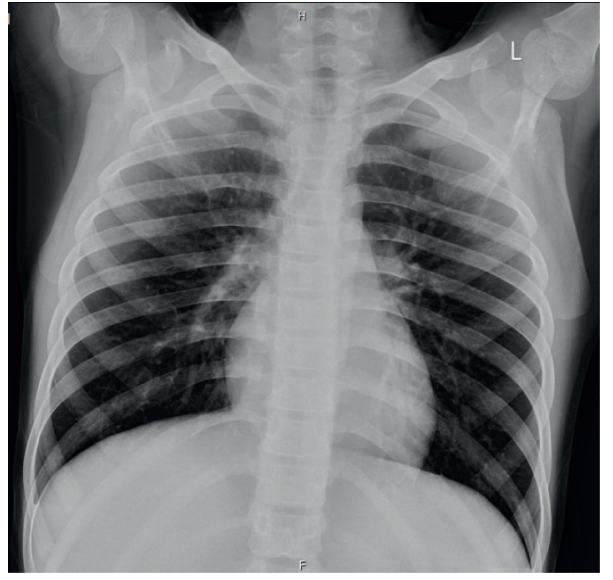

Figure 1: Chest X-ray.

transmembrane protease, serine 2 (TMPRSS2) receptors), as another study by using immunostaining has shown ACE2 is highly expressed in the pancreas; thus, AP in COVID-19 patients could occur due to the direct cytopathic effect of local virus replication $[12,13]$. The endothelial location of the ACE2 receptors and response to SARS-CoV-2 infection may cause increased thrombophilia in pancreas vessels in COVID-19 patients, and this vascular thrombosis may lead to AP [14]. Also, AP may be caused by the indirect effect of the harmful systemic immune response induced by SARS-CoV-2 infection [12]. A certain association between COVID-19 and AP has not been proved yet so we recommend further research to be conducted to evaluate any possible relation. A study reported serum lipase level rising in COVID-19 patients without AP symptoms [15]. Therefore, we suggest that AP diagnosis should be based on Atlanta criteria and not only on the serum lipase level. In addition, the serum lipase level may not be specific and can rise in other diseases and conditions including increased gut permeability (diarrhea) with SARS-CoV-2 infection [16, 17]. 


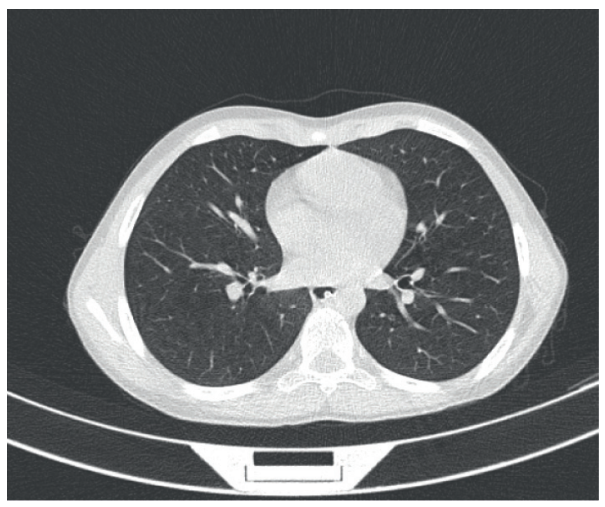

Figure 2: Chest CT.

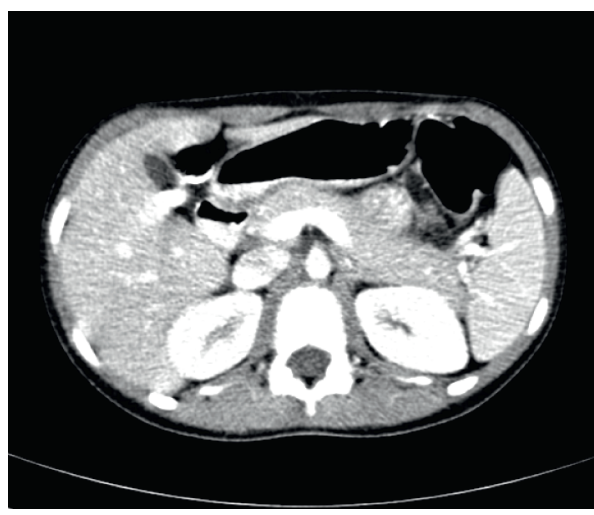

(a)

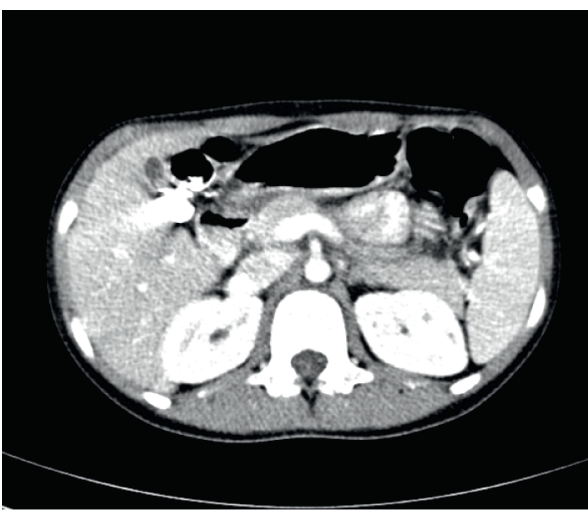

(b)

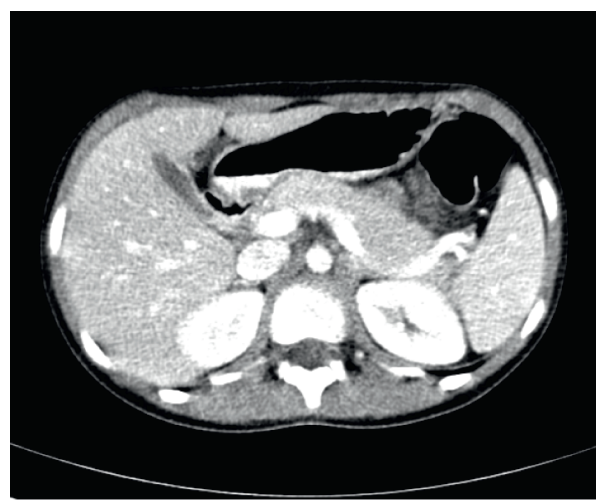

(c)

Figure 3: Abdomen CT.

Considering the possible association between COVID-19 and $\mathrm{AP}$, we suggest full personal protective equipment (PPE) in contact with patients with AP symptoms and also in COVID-19 recognition test if possible.

\section{Abbreviations}

SARS-CoV- Severe acute respiratory syndrome 2: $\quad$ coronavirus-2

COVID-19: Coronavirus disease-2019.

\section{Consent}

No written consent has been obtained from the patient as there are no patient identifiable data included in this case report.

\section{Conflicts of Interest}

The authors declare no conflicts of interest. 


\section{References}

[1] N. Zhu, D. Zhang, W. Wang et al., "A novel coronavirus from patients with pneumonia in China, 2019," New England Journal of Medicine, vol. 382, no. 8, pp. 727-733, 2020.

[2] R. Castagnoli, M. Votto, A. Licari et al., "Severe acute respiratory syndrome coronavirus 2 (SARS-CoV-2) infection in children and adolescents: a systematic review," JAMA Pediatrics, vol. 24, 2020.

[3] A. Hoang, K. Chorath, A. Moreira et al., "COVID-19 in 7780 pediatric patients: a systematic review," Clinical Medicine, vol. 24, 2020.

[4] J. R. DeBanto, P. S. Goday, M. R. A. Pedroso et al., "Acute pancreatitis in children," The American Journal of Gastroenterology, vol. 97, no. 7, pp. 1726-1731, 2002.

[5] P. Rawla, S. S. Bandaru, and A. R. Vellipuram, "Review of infectious etiology of acute pancreatitis," Gastroenterology Research, vol. 10, no. 3, pp. 153-158, 2017.

[6] L. Kottanattu, S. A. G. Lava, R. Helbling, G. D. Simonetti, M. G. Bianchetti, and G. P. Milani, "Pancreatitis and cholecystitis in primary acute symptomatic Epstein-Barr virus infection-systematic review of the literature," Journal of Clinical Virology, vol. 82, pp. 51-55, 2016.

[7] D. M. Parenti, W. Steinberg, and P. Kang, "Infectious causes of acute pancreatitis," Pancreas, vol. 13, no. 4, pp. 356-371, 1996.

[8] P. A. Banks, T. L. Bollen, C. Dervenis et al., "Classification of acute pancreatitis-2012: revision of the Atlanta classification and definitions by international consensus," Gut, vol. 62, no. 1, pp. 102-111, 2013.

[9] L. Pan, M. Mu, P. Yang et al., "Clinical characteristics of COVID-19 patients with digestive symptoms in Hubei, China," The American Journal of Gastroenterology, vol. 115, no. 5, pp. 766-773, 2020.

[10] P. Zimmermann and N. Curtis, "Coronavirus infections in children including COVID-19," Pediatric Infectious Disease Journal, vol. 39, no. 5, pp. 355-368, 2020.

[11] T.-H. Chang, J.-L. Wu, and L.-Y. Chang, "Clinical characteristics and diagnostic challenges of pediatric COVID-19: a systematic review and meta-analysis," Journal of the Formosan Medical Association, vol. 119, no. 5, pp. 982-989, 2020.

[12] F. Wang, H. Wang, J. Fan, Y. Zhang, H. Wang, and Q. Zhao, "Pancreatic injury patterns in patients with coronavirus disease 19 pneumonia," Gastroenterology, vol. 159, no. 1, pp. 367-370, 2020.

[13] J.-K. Yang, S.-S. Lin, X.-J. Ji, and L.-M. Guo, "Binding of SARS coronavirus to its receptor damages islets and causes acute diabetes," Acta Diabetologica, vol. 47, no. 3, pp. 193-199, 2010.

[14] B. Bikdeli, M. V. Madhavan, D. Jimenez et al., "COVID-19 and thrombotic or thromboembolic disease: implications for prevention, antithrombotic therapy, and follow-up," Journal of the American College of Cardiology, vol. 75, no. 23, pp. 2950-2973, 2020.

[15] J. McNabb-Baltar, D. X. Jin, A. S. Grover et al., "Lipase elevation in patients with COVID-19," The American journal of gastroenterology, 2020.

[16] H. Zhang, Z. Kang, H. Gong et al., "Digestive system is a potential route of COVID-19: an analysis of single-cell coexpression pattern of key proteins in viral entry process," Gut, vol. 69, no. 6, pp. 1010-1018, 2020.

[17] D. X. Jin, A. L. Yang, S. L. Suleiman, J. McNabb-Baltar, and P. A. Banks, "Marked serum lipase elevations are associated with longer hospitalizations in patients with non-pancreatic hyperlipasemia," Gastroenterology, vol. 156, no. 6, 2019. 